\title{
Gauge-Higgs Unification and LHC/ILC
}

\author{
Yutaka Hosotani* \\ Department of Physics, Osaka University, \\ Toyonaka, Osaka 560-0043, Japan \\ *E-mail: hosotani@phys.sci.osaka-u.ac.jp
}

\begin{abstract}
In the gauge-Higgs unification scenario the 4D Higgs field is identified with the zero mode of the extra-dimensional component of gauge potentials. The mass of the Higgs particle in the unification in the Randall-Sundrum warped spacetime is predicted to be in the range $100 \mathrm{GeV}-300 \mathrm{GeV}$. The $W W Z$ gauge couplings remains almost universal as in the standard model, but substantial deviation results for the Higgs couplings. The $W W H$ and $Z Z H$ couplings are suppressed by a factor $\cos \theta_{H}$ from the values in the standard model, where $\theta_{H}$ is the Yang-Mills AB phase along the fifth dimension. These can be tested at LHC and ILC.
\end{abstract}

Keywords: Gauge-Higgs unification, Hosotani mechanism

\section{Origin of the Higgs boson}

There is one particle missing in the standard model of electroweak interactions. It is the Higgs boson. The Higgs boson must exist, either as an elementary particle or as a composite particle. The electroweak unification is possible, only if there is something which breaks $S U(2)_{L} \times U(1)_{Y}$ symmetry to $U(1)_{E M}$ symmetry. In the standard model the Higgs boson, whose potential is such that the electroweak symmetry is spontaneously broken, gives masses to $W$ and $Z$ bosons. It also gives quarks and leptons masses through Yukawa couplings.

The standard model seems economical, but it hides dirty secret. Physics ought to be based on simple principles, but there seems no good principle for the Higgs sector. As a result the standard model is afflicted with many arbitrary parameters. There have been many proposals. Technicolor theory views the Higgs boson as a composite state resulting from strong technicolor interactions. Supersymmetry (SUSY) is a leading candidate beyond the standard model which cures the gauge hierarchy problem. However, the situation concerning a large number of arbitrary parameters becomes worse in the minimal supersymmetric standard model. There are other proposals 
such as the little Higgs theory and the Higgsless theory as well.

In this article I would like to argue that the Higgs field is "clean". The Higgs field is a part of gauge fields in higher dimensions, the Higgs sector being controlled by the gauge principle. The difference between the Higgs particle and gauge bosons originates from the structure of the extradimensional space. The scenario is called the gauge-Higgs unification.

The gauge-Higgs unification scenario can be tested at LHC and ILC. ${ }^{1-4}$ It predicts that the mass of the Higgs particle is around $100 \mathrm{GeV}-300$ $\mathrm{GeV}$, exactly in the energy region where LHC can explore. Further the couplings of the Higgs particle to the $\mathrm{W}$ and $\mathrm{Z}$ bosons, and also to quarks and leptons are substantially reduced compared with those in the standard model. Thus the Higgs experiments at LHC may uncover the origin of the Higgs particle, and disclose the existence of extra dimensions.

\section{Old gauge-Higgs unification}

The idea of the gauge-Higgs unification is very old. ${ }^{5-7}$ In the Kaluza-Klein theory the gravity in five dimensional spacetime of topology $M^{4} \times S^{1}$ unifies the four-dimensional gravity with the electromagnetism. The part of the metric, $g_{\mu 5}(\mu=0,1,2,3)$, contains the $4 \mathrm{D}$ vector potential $A_{\mu}$ in the electromagnetism. In the gauge-Higgs unification one considers gauge theory, instead of gravity, in higher dimensional spacetime. Extra-dimensional components, $A_{y_{j}}$, of gauge potentials transform as $4 \mathrm{D}$ scalars under $4 \mathrm{D}$ Lorentz transformations. The 4D Higgs field is identified with a low energy mode of $A_{y_{j}}$. The Higgs field becomes a part of gauge fields.

This scenario was proposed by Fairlie and by Forgacs and Manton in 1979. They tried to achieve unification by restricting configurations of gauge fields in extra dimensions with symmetry ansatz. In ref. 7 Manton considered gauge theory with gauge group $\mathcal{G}$ defined on $M^{4} \times S^{2}$. It is assumed that only spherically symmetric configurations are allowed and gauge fields have non-vanishing flux (field strengths) on $S^{2}$. Further it is demanded that the gauge group $\mathcal{G}$ breaks down to $S U(2)_{L} \times U(1)_{Y}$ by non-vanishing flux. There appears a Higgs doublet as a low energy mode of $A_{y_{j}}$. Quite amazingly the Higgs doublet turns out to have a negative mass squared so that the symmetry further breaks down to $U(1)_{E M}$.

There are two parameters; the radius $R$ of $S^{2}$ and the gauge coupling $g_{6}$ in the six-dimensional spacetime. These two parameters are fixed by the Fermi constant and the four-dimensional $S U(2)_{L}$ gauge coupling $g . m_{W}$, $m_{Z}$, and $m_{H}$ are determined as functions of $g_{6}$ and $R$. The Weinberg angle $\theta_{W}$ is determined by the gauge group only. There are three gauge groups 
Table 1. Spectrum in the gauge-Higgs unification model $^{7}$ by Manton.

\begin{tabular}{|c|c|c|c|c|}
\hline $\mathcal{G}$ & $\sin ^{2} \theta_{W}$ & $m_{W}$ & $m_{Z}$ & $m_{H}$ \\
\hline$S U(3)$ & $3 / 4$ & $44 \mathrm{GeV}$ & $88 \mathrm{GeV}$ & $88 \mathrm{GeV}$ \\
\hline$O(5)$ & $1 / 2$ & $54 \mathrm{GeV}$ & $76 \mathrm{GeV}$ & $76 \mathrm{GeV}$ \\
\hline$G_{2}$ & $1 / 4$ & $76 \mathrm{GeV}$ & $88 \mathrm{GeV}$ & $88 \mathrm{GeV}$ \\
\hline
\end{tabular}

which satisfy the above requirements. The result is summarized in Table 1 .

The unification is achieved and the Higgs mass is predicted, though numerical values are not realistic. There are generic problems in this scheme. First, the mass $m_{Z}$ is $\sim 1 / R$. In other words, it necessarily predicts a too small Kaluza-Klein scale $1 / R$. Secondly, and more importantly, there is no justification for the ansatz of non-vanishing flux. The restriction to spherically symmetric configurations is not justified either.

\section{New gauge-Higgs unification}

There is a better way of achieving gauge-Higgs unification. The key is to consider gauge theory in a non-simply connected spacetime. It utilizes the Hosotani mechanism. ${ }^{8-11}$

\subsection{Yang-Mills AB phase $\theta_{H}$}

When the space is not simply connected, a configuration of vanishing field strengths does not necessarily mean trivial. The phenomenon is called the Aharonov-Bohm (AB) effect in quantum mechanics. Consider $S U(N)$ gauge theory on $M^{4} \times S^{1}$ with coordinates $\left(x^{\mu}, y\right)$, and impose periodic boundary conditions $A_{M}(x, y+2 \pi R)=A_{M}(x, y)$. A configuration $A_{y}(x, y)=$ constant gives $F_{M N}=0$, but gives

$$
W \equiv P \exp \left\{i g \int_{0}^{2 \pi R} d y A_{y}\right\}=U\left(\begin{array}{ccc}
e^{i \theta_{1}} & & \\
& \ddots & \\
& & e^{i \theta_{N}}
\end{array}\right) U^{\dagger}
$$

where $U^{\dagger}=U^{-1}$ and $\sum_{j=1}^{N} \theta_{J}=0(\bmod 2 \pi) . \theta_{j}$ 's are Yang-Mills AB phases in the theory, denoted collectively as $\theta_{H}$. They cannot be eliminated by gauge transformations preserving the boundary conditions.

Classical vacua are degenerate. Yang-Mills AB phases $\theta_{H}$ label flat directions of the classical potential. The degeneracy is lifted at the quantum 
level. The mass spectrum $\left\{m_{n}\right\}$ of various fields depends on $\theta_{H}$. The effective potential $V_{\text {eff }}\left(\theta_{H}\right)$ is given at the one loop level by

$$
V_{\text {eff }}\left(\theta_{H}\right)=\sum \mp \frac{i}{2} \int \frac{d^{4} p}{(2 \pi)^{4}} \sum_{n} \ln \left\{-p^{2}+m_{n}^{2}\left(\theta_{H}\right)\right\} .
$$

The value of $\theta_{H}$ is determined by the location of the global minimum of $V_{\text {eff }}\left(\theta_{H}\right)$.

\subsection{Dynamical gauge symmetry breaking}

Once the matter content is specified, the effective potential is determined and so is the value of $\theta_{H}$ in the true vacuum. Suppose that all fields are periodic so that the boundary conditions are $S U(N)$ symmetric. If $\theta_{H} \neq 0$, the symmetry breaks down to a subgroup of $S U(N)$ in general. In other words we have dynamical gauge symmetry breaking.

Take $S U(3)$ as an example. In a pure gauge theory the global minima are located at $\theta_{1}=\theta_{2}=\theta_{3}=0, \frac{2}{3} \pi, \frac{4}{3} \pi$. The $S U(3)$ symmetry is unbroken. Add periodic fermions in the fundamental representation. Then the global minimum is given by $\theta_{1}=\theta_{2}=\theta_{3}=0$, the $S U(3)$ symmetry remaining unbroken. If one has, instead, a periodic fermion in the addjoint representation, then the global minima are found at $\left(\theta_{1}, \theta_{2}, \theta_{3}\right)=\left(0, \frac{2}{3} \pi,-\frac{2}{3} \pi\right)$ and its permutations. The $S U(3)$ symmetry breaks down to $U(1) \times U(1)$. These results are tabulated in Table 2. Dynamical gauge symmetry breaking occurs quite naturally. It involves no fine tuning. ${ }^{12}$

Instead of periodic boundary conditions, one might impose more general twisted boundary conditions. For instance, one can impose $A_{M}(x, y+$ $2 \pi R)=\Omega A_{M}(x, y) \Omega^{\dagger}(\Omega \in S U(N))$. It can be shown that on $M^{4} \times S^{1}$ physics does not depend on the choice of $\Omega$, thanks to dynamics of YangMills $\mathrm{AB}$ phases $\theta_{H}$. On orbifolds such as $M^{4} \times\left(S^{1} / Z_{2}\right)$ and the RandallSundrum warped spacetime there appear a finite number of inequivalent

Table 2. Dynamical gauge symmetry breaking in $S U(3)$ theory on $M^{4} \times S^{1}$. $N_{\text {fund }}^{F}$ and $N_{\text {add }}^{F}$ denote the number of periodic fermions in the fundamental and addjoint representations, respectively.

\begin{tabular}{|c|c|c|}
\hline$\left(N_{\text {fund }}^{F}, N_{\text {add }}^{F}\right)$ & global minima $\left(\theta_{1}^{\min }, \theta_{2}^{\min }, \theta_{3}^{\min }\right)$ & residual symmetry \\
\hline$(0,0)$ & $(0,0,0),\left( \pm \frac{2}{3} \pi, \pm \frac{2}{3} \pi, \pm \frac{2}{3} \pi\right)$ & $S U(3)$ \\
\hline$(n, 0)$ & $(0,0,0)$ & $S U(3)$ \\
\hline$(0, n)$ & $\left(0,+\frac{2}{3} \pi,-\frac{2}{3} \pi\right)+$ permutations & $U(1) \times U(1)$ \\
\hline$(1,1)$ & $(0, \pi, \pi)+$ permutations & $S U(2) \times U(1)$ \\
\hline
\end{tabular}


sets of boundary conditions..$^{9,13,14}$

\subsection{Finiteness of $V_{\mathrm{eff}}\left(\theta_{H}\right)$ and the Higgs mass}

A mode of four-dimensional fluctuations of Yang-Mills $\mathrm{AB}$ phase $\theta_{H}$ is identified with the $4 \mathrm{D}$ Higgs field in an appropriate setup. Hence $V_{\text {eff }}\left(\theta_{H}\right)$ is directly related to the effective potential for the 4D Higgs field $\varphi_{H}(x)$.

One significant feature is that the $\theta_{H}$-dependent part of $V_{\text {eff }}\left(\theta_{H}\right)$ is finite. The mass squared of the Higgs boson, $m_{H}^{2}$, is essentially the curvature of $V_{\text {eff }}\left(\theta_{H}\right)$ at its global minimum, implying the finiteness of $m_{H}^{2} \cdot{ }^{15}$

The finiteness of $V_{\text {eff }}\left(\theta_{H}\right)$ at the one loop level has been shown explicitly in various models. ${ }^{8,9}$ A general proof goes as follows. ${ }^{12,16}$

First of all large gauge invariance in theory guarantees that $\theta_{H}$ is related to $\theta_{H}+2 \pi$ by a large gauge transformation which preserves the boundary conditions. It implies that $V_{\text {eff }}\left(\theta_{H}+2 \pi\right)=V_{\text {eff }}\left(\theta_{H}\right)$ to all order in perturbation theory. $V_{\text {eff }}\left(\theta_{H}\right)$ can be expanded in a Fourier series; $V_{\text {eff }}\left(\theta_{H}\right)=\sum_{n} a_{n}^{V} e^{i n \theta_{H}}$.

The one loop effective potential is given by (2). In flat space $S^{1}$ $m_{n}\left(\theta_{H}\right)=\left(n+\ell \theta_{H} / 2 \pi+\alpha\right) m_{K K}$. Here the Kaluza-Klein mass scale $m_{K K}=1 / R$ and $\ell$ is an integer. $\alpha$ is a constant determined by the boundary

condition of each field. It follows that $V_{\text {eff }}^{(k)}\left(\theta_{H}\right)=\partial^{k} V_{\text {eff }}\left(\theta_{H}\right) / \partial \theta_{H}^{k}$ becomes finite for sufficiently large $k$ almost everywhere in $\theta_{H}$. $V_{\text {eff }}^{(k)}\left(\theta_{H}\right)$ can develop infrared divergence at a discrete set of values of $\theta_{H}$ where $m_{n}\left(\theta_{H}\right)$ vanishes, namely at a set of points of measure zero. Hence $n^{k} a_{n}^{V}(n \neq 0)$ becomes finite, implying the finiteness of $V_{\text {eff }}\left(\theta_{H}\right)$ at the one loop level. The argument remains valid in the Randall-Sundrum warped spacetime as $m_{n}\left(\theta_{H}\right) \sim\left(n+\ell \theta_{H} / 2 \pi+\alpha\right) m_{K K}$ for $|n| \gg 1$.

The finiteness seems to hold beyond one loop. It has been shown that $m_{H}^{2}$ in QED in $M^{4} \times S^{1}$ is finite at the two loop level after renormalization in $M^{5} .{ }^{17}$ There is nonperturbative lattice simulation indicating the finiteness as well. ${ }^{18}$

\section{Electroweak interactions}

To apply gauge-Higgs unification scenario to electroweak interactions, several features have to be taken into account. ${ }^{19-29}$ First, the electroweak symmetry is $S U(2)_{L} \times U(1)_{Y}$, which breaks down to $U(1)_{E M}$. The Higgs field $\varphi_{H}$ is an $S U(2)_{L}$ doublet. In the gauge-Higgs unification the Higgs field is a part of gauge fields, or must belong to the adjoint representation of the gauge group $\mathcal{G}$. This means that $\mathcal{G}$ must be larger than $S U(2)_{L} \times U(1)_{Y}$, 
as Fairlie, Forgacs, and Manton originally pointed out. ${ }^{5-7}$

Second, fermion content is chiral. This is highly nontrivial in higher dimensional gauge theory, as a spinor in higher dimensions always contains both right- and left-handed components in four dimensions. The left-right asymmetry in fermion modes at low energies can be induced from nontrivial topology of extra-dimensional space and non-vanishing flux of gauge fields in extra dimensions. There is another, simpler and more powerful, way to have chiral fermions. If the extra-dimensional space is an orbifold, appropriate boundary conditions naturally give rise to chiral fermion content. ${ }^{19,20}$

Let us illustrate how the orbifold structure fits in the gauge-Higgs unification, by taking gauge theory on $M^{4} \times\left(S^{1} / Z_{2}\right)$. The orbifold $M^{4} \times\left(S^{1} / Z_{2}\right)$ is obtained from $M^{4} \times S^{1}$ by identifying $\left(x^{\mu},-y\right)$ and $\left(x^{\mu}, y\right)$. There appear two fixed points (branes) at $y=0$ and $y=\pi R$. We define gauge theory on a covering space of $M^{4} \times\left(S^{1} / Z_{2}\right)$, namely for $-\infty<y<+\infty$, and impose restrictions such that physics is the same at $\left(x^{\mu}, y\right),\left(x^{\mu}, y+2 \pi R\right)$ and $\left(x^{\mu},-y\right)$. The single-valuedness of physics does not necessarily mean that vector potentials $A_{M}$ are single-valued. In gauge theory they may be twisted by global gauge transformation. More explicitly

$$
\left(\begin{array}{c}
A_{\mu} \\
A_{y}
\end{array}\right)\left(x, y_{j}-y\right)=P_{j}\left(\begin{array}{c}
A_{\mu} \\
-A_{y}
\end{array}\right)\left(x, y_{j}+y\right) P_{j}^{-1} \quad(j=0,1)
$$

where $y_{0}=0$ and $y_{1}=\pi R$. Here $P_{j}$ is an element of the gauge group $\mathcal{G}$ satisfying $P_{j}{ }^{2}=1$. When $P_{j} \not \subset 1$, the gauge symmetry is partially broken by the boundary conditions. The physical symmetry, in general, can be different from the residual symmetry given by $\left(P_{0}, P_{1}\right)$. It can be either reduced or enhanced by dynamics of $\theta_{H} \cdot{ }^{10}$

To see how an $S U(2)$ doublet Higgs field emerges, take $\mathcal{G}=S U(3)$ and $P_{0}=P_{1}=\operatorname{diag}(-1,-1,1)$. Then, the orbifold boundary condition (3) implies that $S U(2) \times U(1)$ part of the four-dimensional components $A_{\mu}$ are even under parity at $y=0, \pi R$, which contains zero modes corresponding to $S U(2) \times U(1)$ gauge fields in four dimensions. On the other hand the extra-dimensional component $A_{y}$ has zero modes in the off-diagonal part;

$$
S U(3): A_{y}=\left(\begin{array}{r}
\phi^{+} \\
\phi^{0} \\
\phi^{+*} \phi^{0 *}
\end{array}\right) \quad, \quad \Phi=\left(\begin{array}{l}
\phi^{+} \\
\phi^{0}
\end{array}\right) \text {. }
$$

The zero mode $\Phi$ becomes an $S U(2)$ doublet Higgs field. Take $\mathcal{G}=S O(5)$ and $P_{0}=P_{1}=\operatorname{diag}(-1,-1,-1,-1,1)$ as another example. In this case the $S O(5)$ symmetry breaks down to $S O(4) \simeq S U(2)_{L} \times S U(2)_{R}$. Zero modes 
of $A_{y}$ are

$$
S O(5): A_{y}=\left(\begin{array}{r}
\phi_{1} \\
\phi_{2} \\
\phi_{3} \\
\phi_{4}
\end{array}\right) \quad, \quad \Phi=\left(\begin{array}{l}
\phi_{1}+i \phi_{2} \\
\phi_{4}-i \phi_{3}
\end{array}\right) .
$$

$\Phi$ is an $S U(2)_{L}$ doublet. $\Phi$ is related to the Yang-Mills AB phases by (1).

Chiral fermions naturally emerge. Take $\mathcal{G}=S U(3)$ with $P_{j}$ in (4). Fermions in the fundamental representation of $S U(3)$ obey the boundary condition $\psi\left(x, y_{j}-y\right)=P_{j} \gamma^{5} \psi\left(x, y_{j}+y\right)$ so that $\psi$ is decomposed as

$$
\psi=\left(\begin{array}{cc}
\nu_{L} & \tilde{\nu}_{R} \\
e_{L} & \tilde{e}_{R} \\
\tilde{e}_{L} & e_{R}
\end{array}\right) .
$$

$\nu_{L}, e_{L}$ and $e_{R}$ have zero modes, whereas $\tilde{\nu}_{R}, \tilde{e}_{R}$ and $\tilde{e}_{L}$ do not. Fermion content at low energies is chiral as desired.

In the gauge-Higgs unification scenario the Higgs boson is massless at the tree level. Its mass is generated by radiative corrections. The mass of the Higgs boson is determined by the curvature of the effective potential $V_{\text {eff }}\left(\theta_{H}\right)$ at the minimum. In fig. $1 V_{\text {eff }}\left(\theta_{1}, \theta_{2}\right)$ is displayed in the $U(3) \times U(3)$ model of ref. 25 .
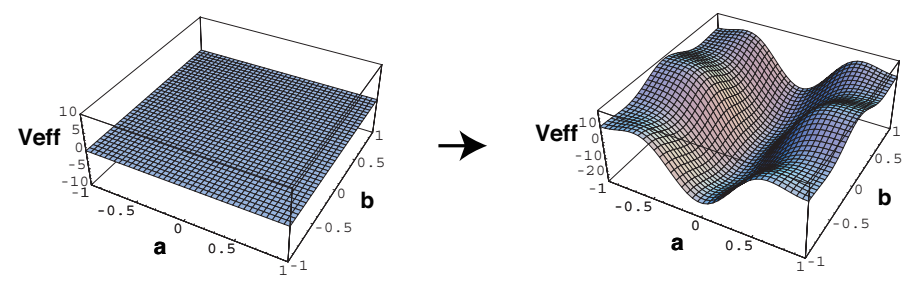

Fig. 1. The effective potential $V_{\text {eff }}\left(\theta_{1}, \theta_{2}\right)$ in the $U(3) \times U(3)$ model in ref. 25 which has two $\theta_{H}$ 's, $\theta_{1}=\pi a$ and $\theta_{2}=\pi b$. $V_{\text {eff }}=0$ at the classical level (in the left figure), but becomes nontrivial at the one loop level (in the right figure).

\section{Difficulties in flat spacetime}

The gauge-Higgs unification scenario in flat spacetime is afflicted with a few intrinsic difficulties. The electroweak symmetry is spontaneously broken by $\theta_{H}$. Non-vanishing $\theta_{H}$ gives rise to non-vanishing masses for $W$ and $Z$ 
bosons. $m_{W}$, for instance, is typically given by

$$
m_{W} \sim \frac{\theta_{H}}{2 \pi} \times \frac{1}{R} \sim \frac{\theta_{H}}{2 \pi} \times m_{K K} .
$$

Here $R$ is the size of the extra-dimensions. Secondly, the effective potential $V_{\text {eff }}\left(\theta_{H}\right)$ is generated at the one-loop level, and therefore is $O\left(\alpha_{W}\right)$ where $\alpha_{W}=g_{W}^{2} / 4 \pi$ is the $S U(2)_{L}$ coupling. The Higgs mass $m_{H}^{2}$ becomes $O\left(\alpha_{W}\right)$ as well. Evaluation of $V_{\text {eff }}$ shows that

$$
m_{H} \sim \sqrt{\alpha_{W}} \times \frac{1}{R} \sim \sqrt{\alpha_{W}} \frac{2 \pi}{\theta_{H}} m_{W} .
$$

The relations (7) and (8) are generic predictions from the gauge-Higgs unification in flat spacetime. Once the value of $\theta_{H}$ is given, $m_{K K}$ and $m_{H}$ are predicted. The value of $\theta_{H}$ is determined from the location of the global minimum of $V_{\text {eff }}\left(\theta_{H}\right)$. It depends on the matter content in the theory. Given standard matter content of quarks and leptons with a minimal set of additional matter, the global minimum of $V_{\text {eff }}\left(\theta_{H}\right)$ is typically located either at $\theta_{H}=0$ or at $\theta_{H}=(.2 \sim .8) \pi$, as confirmed in various models. In the former case the electroweak symmetry remains unbroken. What we want is the latter. In this case $m_{K K} \sim 10 m_{W}$ and $m_{H} \sim 10 \mathrm{GeV}$. One has too low $m_{K K}$ and too small $m_{H}$.

There are two ways to circumvent these difficulties. One way is to arrange the matter content such that small $\theta_{H}$ is obtained. This is possible as discussed by many authors, but requires either many additional fields in higher dimensional representations in $\mathcal{G}$, or fine-tuned cancellations among contributions from various fields. ${ }^{23,24,26}$ Another way is to consider warped (curved) spacetime in extra-dimensions. ${ }^{1-4,30-36}$ Astonishingly the warped spacetime resolves the above problems quite naturally as discussed below.

\section{6. $S O(5) \times U(1)$ unification in warped spacetime}

An attractive model is obtained by considering gauge theory in the RandallSundrum (RS) warped spacetime ${ }^{37-39}$ whose metric is given by

$$
d s^{2}=e^{-2 k \sigma(y)} \eta_{\mu \nu} d x^{\mu} d x^{\nu}+d y^{2}
$$

where $\sigma(y+2 \pi R)=\sigma(y)=\sigma(-y)$ and $\sigma(y)=k|y|$ for $|y| \leq \pi R$. The topology of the spacetime is the same as $M^{4} \times\left(S^{1} / Z_{2}\right)$. The spacetime is an orbifold, with fixed points (branes) at $y=0$ and $\pi R$. It has a negative cosmological constant $\Lambda=-k^{2}$ in the bulk five-dimensional spacetime. The RS spacetime is an anti-de Sitter space sandwiched by the Planck brane 
at $y=0$ and the $\mathrm{TeV}$ brane at $y=\pi R$. At low energies the spacetime resembles four-dimensional Minkowski spacetime.

We consider $S O(5) \times U(1)_{B-L}$ gauge theory ${ }^{31}$ with gauge couplings $g_{A}$ and $g_{B}$ defined in the five-dimensional spacetime (9). We suppose that the structure of the spacetime is determined by physics at the Planck scale and therefore $k=O\left(M_{\mathrm{Pl}}\right)$. With the warp factor $e^{-k \pi R}$ the electroweak scale $m_{W}$ is naturally generated from the Planck scale.

The orbifold boundary conditions for the $S O(5)$ and $U(1)_{B-L}$ gauge fields, $A_{M}$ and $B_{M}$, are given by $P_{0}=P_{1}=\operatorname{diag}(-1,-1,-1,-1,1)$ and $P_{0}=P_{1}=1$ in $(3)$, respectively. With this parity assignment the bulk $S O(5) \times U(1)_{B-L}$ symmetry breaks down to $S O(4) \times U(1)_{B-L}=S U(2)_{L} \times$ $S U(2)_{R} \times U(1)_{B-L}$ on the branes. We further break the symmetry on the Planck brane by imposing the Dirichlet condition on $A_{\mu}^{1_{R}}, A_{\mu}^{2_{R}}$, and $A_{\mu}^{\prime 3_{R}}$ which are even under parity. Here $A_{\mu}^{a_{R}}(a=1,2,3)$ are $S U(2)_{R}$ gauge fields and

$$
A_{\mu}^{\prime 3_{R}}=\frac{g_{A} A_{\mu}^{3_{R}}-g_{B} B_{\mu}}{\sqrt{g_{A}^{2}+g_{B}^{2}}}, A_{\mu}^{Y}=\frac{g_{B} A_{\mu}^{3_{R}}+g_{A} B_{\mu}}{\sqrt{g_{A}^{2}+g_{B}^{2}}} .
$$

$A_{\mu}^{Y}$ obeys the Neumann condition on both branes. As a result the residual symmetry is $S U(2)_{L} \times U(1)_{Y}$. The change of the boundary conditions from Neumann to Dirichlet for $A_{\mu}^{1_{R}}, A_{\mu}^{2_{R}}$, and $A_{\mu}^{\prime 3_{R}}$ is induced by additional dynamics on the Planck brane, and is consistent with the large gauge invariance. ${ }^{3,4,40}$

\subsection{Mass spectrum}

There is one mass scale in the theory, namely $k=O\left(M_{\mathrm{Pl}}\right)$, and a few dimensionless parameters $k \pi R, g_{A} / \sqrt{\pi R}$ and $g_{B} / \sqrt{\pi R}$. The Kaluza-Klein mass scale in the RS spacetime is

$$
m_{K K}=\frac{\pi k}{e^{k \pi R}-1} \sim \begin{cases}\pi k e^{-k \pi R} & \text { for } e^{k \pi R} \gg 1 \\ 1 / R & \text { for } k \rightarrow 0\end{cases}
$$

For $\theta_{H} \neq 0, m_{W}$ and $m_{Z}$ are given by

$$
\begin{aligned}
& m_{W} \sim \sqrt{\frac{k}{\pi R}} e^{-k \pi R} \sin \theta_{H} \\
& m_{Z} \sim \frac{m_{W}}{\cos \theta_{W}} \quad, \quad \sin \theta_{W}=\frac{g_{Y}}{\sqrt{g_{A}^{2}+g_{Y}^{2}}}=\frac{g_{B}}{\sqrt{g_{A}^{2}+2 g_{B}^{2}}} .
\end{aligned}
$$

In a generic situation one has $\sin \theta_{H}=O(1)$. It follows from the relation for $m_{W}$ that the dimensionless parameter $k \pi R \sim 37$ for $k=O\left(M_{\mathrm{Pl}}\right)$. 
Further (11) and (12) imply that

$$
m_{K K} \sim \frac{\pi}{\sin \theta_{H}} \sqrt{k \pi R} m_{W} .
$$

For moderate values $\theta_{H}=(0.2 \sim 0.5) \pi$, the Kaluza-Klein scale turns out $m_{K K}=2.6 \mathrm{TeV} \sim 1.5 \mathrm{TeV}$, which is large enough to be consistent with the current experimental limit. One of the problems in the gauge-Higgs unification scenario in flat spacetime mentioned earlier is solved. In the Randall-Sundrum spacetime there appears an enhancement factor $\sqrt{k \pi R}$.

The mass scale of low energy modes becomes much smaller than the Kaluza-Klein mass scale in the warped spacetime. This can be most clearly seen by examining the mass spectrum as a function of $\theta_{H}$ with various values of $k \pi R$. See fig. 2. $m_{W} / m_{K K}$ has weak dependence on $\theta_{H}$ for $k \pi R=37$ and is much smaller than 1 . In the flat spacetime $m_{W} / m_{K K}$ becomes $O(0.1)$ for $0.1 \pi<\theta_{H}<0.9 \pi$.
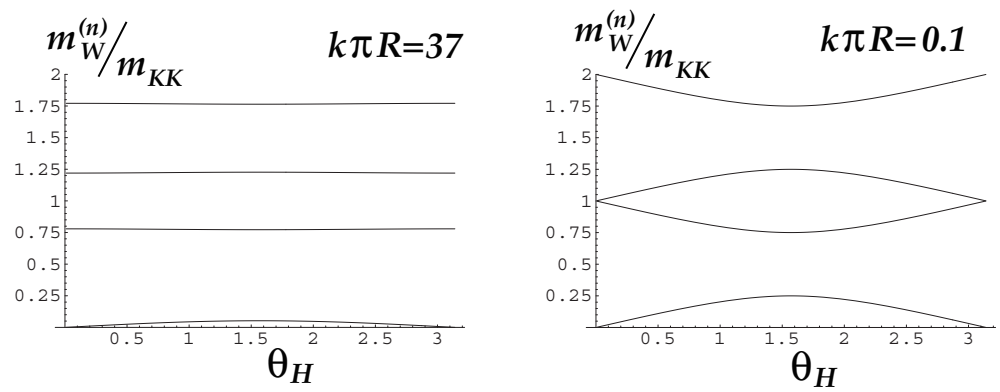

Fig. 2. $m_{W}^{(n)} / m_{K K}(n=0,1,2,3)$ is plotted for $k \pi R=37$ and 0.1 , where the former corresponds to the realistic case, whereas the latter is close to the flat spacetime limit $(k \pi R=0) . m_{W}=m_{W}^{(0)}$.

\subsection{Higgs mass and self-couplings}

The Higgs mass and self-couplings are generated by quantum effects, or by radiative corrections. The $4 \mathrm{D}$ Higgs field $\varphi_{H}(x)$ corresponds to fourdimensional fluctuations of $\theta_{H}$. In the $S O(5) \times U(1)_{B-L}$ model

$$
A_{y}^{\hat{4}}(x, y)=\frac{2 \sqrt{2} k e^{2 k y}}{g_{A}\left(z_{\pi}^{2}-1\right)}\left\{\theta_{H}+\frac{g_{A}}{2} \sqrt{\frac{z_{\pi}^{2}-1}{k}} \varphi_{H}(x)\right\}+\cdots
$$

where $z_{\pi}=e^{k \pi R}$. Thus, the Higgs mass $m_{H}$, for instance, is evaluated from the curvature of $V_{\text {eff }}\left(\theta_{H}\right)$ at the minimum. Notice that $\theta_{H}$ and $\varphi_{H}(x)$ 
appear in the effective potential in the combination of

$$
\begin{cases}\theta_{H}+\frac{\pi g}{\sqrt{2} m_{K K}} \sqrt{\frac{k \pi R}{2}} \varphi_{H}(x) & \text { for } e^{k \pi R} \gg 1, \\ \theta_{H}+\frac{\pi g}{\sqrt{2} m_{K K}} \varphi_{H}(x) & \text { for } k \rightarrow 0,\end{cases}
$$

where the $4 \mathrm{D} S U(2)_{L}$ coupling $g$ is given by $g=g_{A} / \sqrt{\pi R}$. We observe that $k \pi R / 2 \sim 18$ gives various quantities in the warped space an enhancement factor compared with those in flat space.

On general ground the effective potential at one loop is estimated as

$$
V_{\text {eff }}\left(\theta_{H}\right) \sim \frac{3}{128 \pi^{6}} m_{K K}^{4} f\left(\theta_{H}\right)
$$

where $f\left(\theta_{H}\right)=O(1)$ in minimal models. The mass $m_{H}$ and the quartic coupling $\lambda$ (in $\lambda \varphi_{H}^{4} / 4$ !) are evaluated as

$$
m_{H} \sim \sqrt{\frac{3 \alpha_{W}}{32 \pi} f^{(2)}\left(\theta_{H}\right)} \frac{k \pi R}{2} \frac{m_{W}}{\sin \theta_{H}}, \quad \lambda \sim \frac{3 \alpha_{W}^{2}}{32} f^{(4)}\left(\theta_{H}\right)\left(\frac{k \pi R}{2}\right)^{2},
$$

where $\alpha_{W}=g^{2} / 4 \pi$. There is ambiguity in $f^{(2)}, f^{(4)}$ which somewhat depend on detailed content of the model. Inserting typical values $f^{(2)}, f^{(4)} \sim 4$ and $\theta_{H}=(0.1 \sim 0.5) \pi$, one finds that $m_{H}=(90 \sim 290) \mathrm{GeV}$ and $\lambda \sim 0.1$. Although the precise form of $f\left(\theta_{H}\right)$ depends on details of the model, the feature of the enhancement by the factor $k \pi R / 2$ in the $\mathrm{RS}$ spacetime is general. The problem of too small $m_{H}$ in flat spacetime has been solved.

\section{3. $W W Z$ coupling}

When $\theta_{H}=0$, the electroweak symmetry $S U(2)_{L} \times U(1)_{Y}$ remains unbroken. The $S U(2)_{L}$ gauge coupling in four dimensions is given by $g=$ $g_{A} / \sqrt{\pi R}$. All couplings associated with $W$ and $Z$ are determined by the $S U(2)_{L} \times U(1)_{Y}$ gauge principle. When $\theta_{H} \neq 0$, things are not so simple in the gauge-Higgs unification scenario.

With $\theta_{H} \neq 0, S U(2)_{L} \times U(1)_{Y}$ breaks down to $U(1)_{E M}$. In the standard model the $W$ boson resides in the $S U(2)_{L}$ group. In the $S O(5) \times U(1)_{B-L}$ gauge-Higgs unification model, $\theta_{H} \neq 0$ mixes various components of $S U(2)_{L}, S U(2)_{R}$ and $S O(5) / S O(4)$. It also mixes various Kaluza-Klein excited states. The eigenstate $W$ and its wave function are determined by complete diagonalization. This poses an interesting question whether or not the $W W Z$ coupling $g_{W W Z}$, for instance, remains universal as in the standard model. There is no guarantee for that. 
This is an important issue as the LEP2 data on the $W$ pair production rate agrees with the $W W Z$ coupling in the standard model within an error of a few percents. In Table 3 the ratio of $g_{W W Z}$ in the gauge-Higgs unification to that in the standard model is tabulated for various $\theta_{H}$ and $k \pi R$. One sees that for the realistic case $k \pi R \sim 35$, deviation from the standard model is tiny for any values of $\theta_{H}$, whereas in the flat spacetime limit $(k \pi R=0)$ substantial deviation appears for moderate values of $\theta_{H}$.

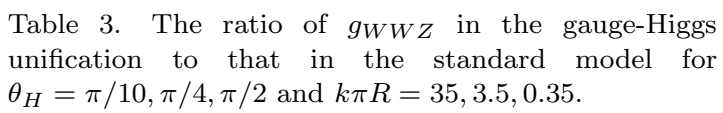

\begin{tabular}{|c|c|c|c|}
\hline & $\theta_{H}=\frac{1}{10} \pi$ & $\frac{1}{4} \pi$ & $\frac{1}{2} \pi$ \\
\hline$k \pi R=35$ & 0.9999987 & 0.999964 & 0.99985 \\
\hline 3.5 & 0.9999078 & 0.996993 & 0.98460 \\
\hline 0.35 & 0.9994990 & 0.979458 & 0.83378 \\
\hline
\end{tabular}

The $W W Z$ coupling remains almost universal in the warped space. The gauge-Higgs unification scenario in the warped space is consistent with the LEP2 data, whereas the scenario in flat space conflicts with the data unless $\theta_{H}$ is sufficiently small.

\section{4. $W W H$ and $Z Z H$ couplings}

There emerges significant deviation from the standard model in various couplings of the Higgs boson. Unlike 4D gauge bosons the 4D Higgs boson is mostly localized near the $\mathrm{TeV}$ brane so that the behavior of wave functions of various fields on the $\mathrm{TeV}$ brane becomes relevant for their couplings to the Higgs boson.

Robust prediction is obtained for the $W W H$ and $Z Z H$ couplings

$$
\lambda_{W W H} H W^{\mu \dagger} W_{\mu}+\frac{1}{2} \lambda_{Z Z H} H Z^{\mu} Z_{\mu} .
$$

The detailed matter content affects the effective potential $V_{\text {eff }}\left(\theta_{H}\right)$, but the couplings $\lambda_{W W H}$ and $\lambda_{Z Z H}$ are determined independent of such details once $\theta_{H}$ is given. One finds that

$$
\lambda_{W W H} \simeq g m_{W} \cdot p_{\mathrm{H}}\left|\cos \theta_{H}\right|, \quad \lambda_{Z Z H} \simeq \frac{g m_{Z}}{\cos \theta_{W}} \cdot p_{\mathrm{H}}\left|\cos \theta_{H}\right|
$$

where $p_{\mathrm{H}} \equiv \operatorname{sgn}\left(\tan \theta_{H}\right)$. Compared with the values in the standard model, both couplings are suppressed by a factor $\cos \theta_{H}$. This result can be used to experimentally test the gauge-Higgs unification scenario. 


\subsection{Yukawa coupling}

Couplings of the Higgs boson to quarks and leptons, Yukawa couplings, are also subject to nontrivial $\theta_{H}$-dependent suppression. The Lagrangian for fermions is given by ${ }^{38,39}$

$$
\begin{gathered}
i \bar{\psi} \Gamma^{a} e_{a}{ }^{M}\left\{\partial_{M}+\frac{1}{8} \omega_{b c M}\left[\Gamma^{b}, \Gamma^{c}\right]-i g_{A} A_{M}-i \frac{g_{B}}{2} \mathcal{Q}_{\mathrm{B}-\mathrm{L}} B_{M}\right\} \psi \\
-c k \epsilon(y) \bar{\psi} \psi+\text { brane interactions }
\end{gathered}
$$

$\mathcal{Q}_{\mathrm{B}-\mathrm{L}}$ is a charge of $U(1)_{B-L}$. The kink mass term $\operatorname{ck} \epsilon(y)$ naturally arises in the Randall-Sundrum spacetime where a dimensionless parameter $c$ for each fermion multiplet plays a crucial role for determining its wave function. There can be "brane interactions" between $\psi$ and additional brane fermion fields defined on one of the branes.

The Higgs coupling to $\psi$ is contained in the gauge interaction involving $A_{y}$. Non-vanishing $\theta_{H}\left(\left\langle A_{y}\right\rangle \neq 0\right)$ induces a finite fermion mass. Although the gauge interaction is universal, the resulting $4 \mathrm{D}$ mass and Yukawa interaction depend on the wave function in the fifth dimension, or on $c$ and the brane interactions. This gives flavor-dependent masses and

Yukawa couplings. In the absence of brane interactions, $c= \pm \frac{1}{2}$ gives a fermion a mass of $O\left(m_{W}\right)$. Light fermions $(e, \mu, \tau, u, d, s, c, b)$ corresponds to $c=(0.6 \sim 0.8)$, whereas a heavy fermion $(t)$ to $c \sim 0.4$. The large hierarchy in the fermion mass spectrum is explained by plain distribution in the parameter $c$.

In the minimal standard model the Yukawa coupling is proportional to the mass of a fermion. In the gauge-Higgs unification scenario this relation is modified. In the absence of brane interactions the Yukawa coupling in the gauge-Higgs unification in the RS spacetime is suppressed by a factor $\cos \theta_{H}$ or $\cos \frac{1}{2} \theta_{H}$ compared with the value in the standard model. To realize the observed spectrum of quarks and leptons, however, one needs to include brane interactions, which in turn affects the relationship between the mass and Yukawa coupling. Although the relationship depends on details of the model, it is expected that it deviates from that in the standard model.

\subsection{Gauge couplings of fermions}

Couplings of quarks and leptons to $W$ and $Z$ also suffer from modification, but the amount of deviation from the standard model turns out tiny. The $\mu-e$ universality in weak interactions played an important role in the development of the theory. In the modern language it says that all left-handed 
leptons and quarks have the same coupling to the $W$ boson. It is dictated by the $S U(2)_{L}$ gauge invariance in four dimensions. In the gauge-Higgs unification, however, the universality is not guaranteed at $\theta_{H} \neq 0$. As explained earlier, non-vanishing $\theta_{H}$ mixes various components in the gauge group and various levels in the Kaluza-Klein tower. This mixing for fermions depends on, say, the kink mass parameter $c$, and therefore is not universal.

For $c>0.6$ wave functions are mostly localized near the Planck brane at $y=0$ so that the $4 \mathrm{D}$ gauge coupling to $W$ becomes almost universal for any values of $\theta_{H}$. Define $r_{\mu}\left(\theta_{H}\right)=g_{\mu}^{W}\left(\theta_{H}\right) / g_{e}^{W}\left(\theta_{H}\right)-1$ where $g_{e}^{W}$ and $g_{\mu}^{W}$ are the gauge $(W)$ couplings of $e$ and $\mu$, respectively. One finds typically that $r_{\mu} \sim-10^{-8}$ for $\theta_{H}=0.5 \pi$. For $\tau, r_{\tau} \sim-2 \times 10^{-6}$. These numbers are well within the experimental limit, being very hard to test in the near future. For top quarks, the deviation becomes bigger $\left(r_{t}(0.5 \pi) \sim-2 \times 10^{-2}\right)$, but is difficult to measure accurately.

\section{Flat v.s. Warped}

Why do we need the warped spacetime rather than flat spacetime? The Randall-Sundrum warped spacetime was originally introduced to naturally explain the hierarchy between the Planck scale and weak scale. When applied to the gauge-Higgs unification, there are more benefits.

See Table 4. Both Higgs mass and Kaluza-Klein mass scale turn out too small in flat space for moderate values of $\theta_{H}$. The $\rho$ parameter deviates from 1 even at the tree level and the $W W Z$ coupling deviates from the value in the standard model in falt space. All these problems are resolved in the Randall-Sundrum warped space. Besides the observed fermion spectrum can be explained without any fine tuning of the parameters.

All of them indicate that having the Randall-Sundrum warped spacetime as background is not just an accident, but have a deeper reason. In this regard the holographic interpretation of the model in the AdS/CFT correspondence is very suggestive as explored by many authors.

\section{Conclusion}

The prospect of the gauge-Higgs unification in the warped spacetime is bright. The Higgs field is identified with the Yang-Mills AB phase in the extra dimension. It gives definitive prediction in the Higgs couplings, which can be tested at LHC and ILC. The model has not been completed yet. The most urgent task includes to pin down additional brane interactions for fermions so that the observed quark-lepton mass spectrum and the CKM 
Table 4. Comparison of the gauge-Higgs unification in the $S O(5) \times U(1)$ model in the flat spacetime $M^{4} \times\left(S^{1} / Z_{2}\right)$ and in the Randall-Sundrum warped spacetime. $\theta_{H}=(0.1 \sim 0.5) \pi . k \pi R=37$ for the RS spacetime. The estimate of $m_{H}$ has ambiguity in $f^{(2)}\left(\theta_{H}\right)$ in (17).

\begin{tabular}{|c|c|c|}
\hline Background spacetime & $M^{4} \times\left(S^{1} / Z_{2}\right)$ & Randall-Sundrum \\
\hline Higgs mass $m_{H}$ & $3 \sim 16 \mathrm{GeV}$ & $100 \sim 300 \mathrm{GeV}$ \\
\hline KK mass scale $m_{K K}$ & $0.3 \sim 1.1 \mathrm{TeV}$ & $1.5 \sim 5.0 \mathrm{TeV}$ \\
\hline $\sin \theta_{W}, \rho$ & deviation from SM & OK \\
\hline$W W Z$ coupling & deviation from SM & OK (almost universal) \\
\hline$W W H$ coupling & - & suppressed by $\cos \theta_{H}$ \\
\hline$Z Z H$ coupling & - & suppressed by $\cos \theta_{H}$ \\
\hline Quark-lepton spectrum & fine tuning necessary & natural hierarchy \\
\hline Yukawa couplings & - & generally suppressed \\
\hline
\end{tabular}

and MNS mixing matrices are reproduced.

\section{Acknowledgements}

This work was supported in part by Scientific Grants from the Ministry of Education and Science, Grant No. 17540257, Grant No. 13135215 and Grant No. 18204024. The author would like to thank the Aspen Center for Physics for its hospitality where a part of this work was performed.

\section{References}

1. Y. Hosotani and M. Mabe, Phys. Lett. B615 (2005) 257.

2. Y. Hosotani, S. Noda, Y. Sakamura and S. Shimasaki, Phys. Rev. D73 (2006) 096006.

3. Y. Sakamura and Y. Hosotani, Phys. Lett. B645 (2007) 442.

4. Y. Hosotani and Y. Sakamura, hep-ph/0703212.

5. D.B. Fairlie, Phys. Lett. B82 (1979) 97; J. Phys. G5 (1979) L55.

6. P. Forgacs and N. Manton, Comm. Math. Phys. 72 (1980) 15.

7. N. Manton, Nucl. Phys. B158 (1979) 141.

8. Y. Hosotani, Phys. Lett. B126 (1983) 309.

9. Y. Hosotani, Ann. Phys. (N.Y.) 190 (1989) 233.

10. N. Haba, M. Harada, Y. Hosotani and Y. Kawamura, Nucl. Phys. B657 (2003) 169; Erratum, ibid. B669 (2003) 381.

11. A. Hebecker and J. March-Russell, Nucl. Phys. B625 (2002) 128.

L. Hall, H. Murayama, and Y. Nomura, Nucl. Phys. B645 (2002) 85.

12. Y. Hosotani, in the Proceedings of "Dynamical Symmetry Breaking", ed. 
M. Harada and K. Yamawaki (Nagoya University, 2004), p. 17. (hep$\mathrm{ph} / 0504272)$.

13. N. Haba, Y. Hosotani and Y. Kawamura, Prog. Theoret. Phys. 111 (2004) 265.

14. Y. Hosotani, in "Strong Coupling Gauge Theories and Effective Field Theories", ed. M. Harada, Y. Kikukawa and K. Yamawaki (World Scientific 2003), p. 234. (hep-ph/0303066).

15. H. Hatanaka, T. Inami and C.S. Lim, Mod. Phys. Lett. A13 (1998) 2601.

16. Y. Hosotani, hep-ph/0607064.

17. N. Maru and T. Yamashita, NPB754 (2006) 127.

18. N. Irges and F. Knechtli, Nucl. Phys. B719 (2005) 121; hep-lat/0604006; hep-lat/0609045.

19. A. Pomarol and M. Quiros, Phys. Lett. B438 (1998) 255.

20. I. Antoniadis, K. Benakli and M. Quiros, New. J. Phys.3 (2001) 20.

21. C. Csaki, C. Grojean and H. Murayama, Phys. Rev. D67 (2003) 085012.

22. L.J. Hall, Y. Nomura and D. Smith, Nucl. Phys. B639 (2002) 307;

G. Burdman and Y. Nomura, Nucl. Phys. B656 (2003) 3;

C.A. Scrucca, M. Serone, L. Silvestrini and A. Wulzer, JHEP 0402 (2004) 49.

23. N. Haba, Y. Hosotani, Y. Kawamura and T. Yamashita, Phys. Rev. D70 (2004) 015010.

24. N. Haba, K. Takenaga, and T. Yamashita, Phys. Lett. B615 (2005) 247.

25. Y. Hosotani, S. Noda and K. Takenaga, Phys. Lett. B607 (2005) 276.

26. G. Cacciapaglia, C. Csaki and S.C. Park, JHEP 0603 (2006) 099.

27. G. Panico, M. Serone and A. Wulzer, Nucl. Phys. B739 (2006) 186.

28. B. Grzadkowski and J. Wudka, PRL97 (2006) 211602.

29. C.S. Lim and N. Maru, hep-ph/0703017.

30. R. Contino, Y. Nomura and A. Pomarol, Nucl. Phys. B671 (2003) 148.

31. K. Agashe, R. Contino and A. Pomarol, Nucl. Phys. B719 (2005) 165.

32. K. Oda and A. Weiler, Phys. Lett. B606 (2005) 408.

33. M. Carena, E. Ponton, J. Santiago and C.E.M. Wagner, Nucl. Phys. B759 (2006) 202; hep-ph/0701055.

34. T. Gherghetta, hep-ph/0601213.

35. A. Falkowski, Phys. Rev. D75 (2007) 025017.

36. R. Contino, T. Kramer, M. Son and R. Sundrum, hep-ph/0612180.

37. L. Randall and R. Sundrum, Phys. Rev. Lett. 83 (1999) 3370.

38. T. Gherghetta and A. Pomarol, Nucl. Phys. B586 (2000) 141.

39. S. Chang, J. Hisano, H. Nakano, N. Okada and M. Yamaguchi, Phys. Rev. D62 (2000) 084025.

40. N. Sakai and N. Uekusa, hep-th/0604121. 